\title{
Multidisciplinary treatment system for bone metastases for early diagnosis, treatment and prevention of malignant spinal cord compression
}

\author{
EIJI NAKATA ${ }^{1,2}$, SHINSUKE SUGIHARA ${ }^{2}$, YOSHIFUMI SUGAWARA ${ }^{3}$, RYUICHI NAKAHARA ${ }^{1}$, \\ TAKAYUKI FURUMATSU ${ }^{1}$, TOMONORI TETSUNAGA ${ }^{1}$, TOSHIYUKI KUNISADA ${ }^{1}$, \\ KAZUO NAKANISHI $^{4}$, YOSHITERU AKEZAKI ${ }^{5}$ and TOSHIFUMI OZAKI ${ }^{1}$ \\ ${ }^{1}$ Department of Orthopedic Surgery, Okayama University Hospital, Okayama 700-8558; \\ Departments of ${ }^{2}$ Orthopedic Surgery and ${ }^{3}$ Radiology, Shikoku Cancer Center, Matsuyama, Ehime 791-0280; \\ ${ }^{4}$ Department of Orthopedic Surgery, Kawasaki Medical School Hospital, Okayama 701-0192; \\ ${ }^{5}$ Division of Physical Therapy, Kochi Professional University of Rehabilitation, Tosa, Kochi 781-1102, Japan
}

Received July 15, 2019; Accepted November 29, 2019

DOI: $10.3892 / 01.2020 .11415$

\begin{abstract}
Malignant spinal cord compression (MSCC) is a serious complication of cancers. The present study aimed to establish a multidisciplinary treatment system for urgent magnetic resonance imaging (MRI) and referral to orthopedists in order to prevent neurological deficits caused by MSCC. In the present study, the extent to which this system achieved early diagnosis and treatment and prevented MSCC-caused neurological deficits was examined. The records from patients with neurological deficits caused by MSCC before (between April 2007 and March 2012; group A) and after (between April 2012 and March 2017; group B) the establishment of the multidisciplinary system at the Shikoku Cancer Center (Ehime, Japan) were retrospectively evaluated. The numbers of patients with neurological deficits were 38 and 7 in groups A and B, respectively. All patients received radiotherapy. The incidence of neurological deficits was 13.2 and $3.4 \%$ in groups A and B, respectively $(\mathrm{P}<0.001)$. The proportion of patients with improvement in the severity of neurological deficits was 5.3 and $28.6 \%$ in groups $\mathrm{A}$ and $\mathrm{B}$, respectively $(\mathrm{P}<0.001)$. The interval between physicians' recognition of a neurological deficit and MRI and the start of treatment, the number of cases, and the severity of neurological deficits were evaluated in groups A and B. The median interval between recognition of a neurological deficit by physicians and MRI was 3 and 0 days in groups A and B, respectively $(\mathrm{P}<0.001)$. The median interval between physicians' recognition of a neurological deficit and the start of treatment
\end{abstract}

Correspondence to: Dr Eiji Nakata, Department of Orthopedic Surgery, Okayama University Hospital, 2-5-1 Shikata-cho, Okayama 700-8558, Japan

E-mail: eijinakata8522@yahoo.co.jp

Key words: bone metastasis, multidisciplinary treatment, skeletal-related event, malignant spinal cord compression, neurological deficit was 3 and 0 days in groups $\mathrm{A}$ and $\mathrm{B}$, respectively $(\mathrm{P}<0.001)$. By using a multidisciplinary treatment system, the incidence and severity of neurological deficits following treatment were significantly improved. Therefore, the multidisciplinary treatment system used in the present study may be useful for early diagnosis, treatment and prevention of MSCC in patients with bone metastases.

\section{Introduction}

Patients with bone metastases commonly present with spinal metastases, and $60-70 \%$ of patients with advanced cancer develop spinal metastases during the progression of the disease $(1,2)$. Spinal metastases are asymptomatic when their size and number are small; however, they gradually progress and frequently lead to skeletal-related events, including radiation therapy (RT) due to painful vertebral metastases, vertebral body fracture and malignant spinal cord compression (MSCC) (1). In patients without a neurological deficit, the pain from vertebral bone metastases can be controlled by RT in most cases (3). Our previous study reported that disappearance of pain and re-ossification were achieved at 3 months following RT in patients with painful vertebral bone metastases without paralysis (4). In addition, no patients presented with pain that was difficult to control with conventional radiotherapy or required surgery. Therefore, it was concluded that early diagnosis and treatment of bone metastases can lead to improved results $(3,4)$.

MSCC is one of the most serious complications of cancer, and $2.5-5 \%$ of patients with advanced cancer develop MSCC $(1,2)$. MSCC can occur with all types of cancer; however, MSCC is most frequently observed in patients with breast, prostate or lung cancer $(5,6)$.

In MSCC, the spinal cord is damaged by compression or vascular compromise due to tumor growth, which leads to neurological dysfunction $(5,6)$. MSCC is an oncological emergency since it causes motor weakness, sensory deficits, walk disturbance, and urinary and bowel dysfunction that markedly impair the quality of life (7-9). 
The importance of local control of bone metastases is increasing since patients with advanced cancer live longer due to recent advances in systemic therapies. Therefore, early diagnosis and treatment of MSCC, and a prophylactic approach for MSCC are crucial. However, several days may pass between the diagnosis and treatment of the neurological deficit ('Doctor's Delay'), and the severity of the neurological deficit is often advanced at the time of referral to orthopedists and it is frequently difficult to improve the neurological deficit $(8,9)$.

In most cases, back pain in the metastatic vertebral bone is the primary symptom preceding neurological dysfunction $(5,10)$. Therefore, guidelines for bone metastases recommend that clinicians watch for early suspicious signs of MSCC and make a prompt diagnosis by spine magnetic resonance imaging (MRI) $(5,10)$. According to the MSCC guidelines of the National Institute for Health and Clinical Excellence in the United Kingdom, spine MRI is recommended as soon as possible when symptoms of MSCC, including back pain and neurological deficit, appear in patients with a history of cancer (5).

Doctors should communicate and share information regarding bone metastases in order to facilitate their treatment, since bone metastases occur in various types of cancer. It is crucial to establish a multidisciplinary treatment system for the management of bone metastases in hospitals (11-18). Recently, numerous institutions in Japan have organized cancer boards, including doctors, nurses and other paramedical staff, in order to focus on the management of bone metastases (19). However, the actual benefit of these cancer boards has not been clearly identified.

To the best of our knowledge, no study has reported a decreased incidence of neurological deficits due to spinal metastases when a multidisciplinary treatment system was implemented. Therefore, the present study investigated the change in the number of patients with neurological deficits before and after the establishment of a multidisciplinary treatment system for bone metastases. Furthermore, the differences in the time intervals to MRI and RT before and after the establishment of the multidisciplinary treatment system were examined.

\section{Materials and methods}

Patients and methods. The file records of 45 patients ( 24 male and 21 female; age, 30-82 years) who were followed-up in Shikoku Cancer Center (Matsuyama, Japan) for advanced cancer, presented with bone metastases and developed neurological deficits due to MSCC before (between April 2007 and March 2012; group A) and after (between April 2012 and March 2017; group B) the establishment of the multidisciplinary treatment system at the Shikoku Cancer Center were retrospectively evaluated. Patients were excluded if they had paralysis at their first presentation or the origin of the neurological deficit was the brain.

In 2012, a multidisciplinary treatment system for bone metastases was established at the Shikoku Cancer Center to prevent neurological deficits caused by vertebral bone metastases. Patients with bone metastases were identified by radiologists via regular computed tomography (CT). Contrast-enhanced CT was performed routinely.
The radiologists paid attention to destruction of the vertebral column cortex since this indicates the existence of MSCC (Fig. 1A-D). When radiologists confirmed MSCC or severe vertebral body destruction (destruction of $>1 / 3$ of a vertebral body) during image interpretation, they described these findings in their reports and wrote comments recommending referral of the patients to orthopedists for a consultation or to receive RT. Physicians could refer patients to orthopedists or radiotherapists after reading these comments.

The orthopedists met with the patients following referral by physicians. They evaluated the severity of the neurological deficit (motor function) according to the Frankel classification (20). Furthermore, they determined whether the origin of the neurological deficit was the brain, the spinal cord or other peripheral nerves. They also evaluated the spinal instability in order to decide whether conservative treatment (with or without orthosis) or surgery was required (3).

Additionally, physicians were advised to consider the existence of back pain in patients with vertebral bone metastases due to the associated-risk of developing paralysis. A rapid referral system was established to provide urgent access to MRI scanning, referral to orthopedists and administration of RT for the urgent management of neurological deficits caused by MSCC.

It was difficult to determine the precise number of patients with bone metastases. Therefore, the rate of patients with neurological deficits among those who received RT for vertebral metastases was investigated. The numbers of patients who received RT before and after the establishment of the system were 286 and 206, respectively (Table SI). The primary tumor sites of patients before the establishment of the system were lung (94 patients), breast (67), prostate (27), stomach (17) colorectum (12) and other (69). The primary tumor sites of patients after the establishment of the system were lung (65 patients), breast (46), prostate (34), colorectum (13), stomach (8) and other (40). The severity of neurological deficits at the time of their recognition by physicians and at the time of treatment in groups A and B were evaluated using the Frankel classification (20).

The time intervals between the recognition of neurological deficits by physicians and MRI scanning and the start of treatment were also investigated. Furthermore, the rate of referral to orthopedists for neurological deficits before treatment and the use of bone-modifying agents (BMAs), such as denosumab or zoledronic acid were evaluated in groups $\mathrm{A}$ and $\mathrm{B}$.

The present study was approved by the Ethical Review Board of Shikoku Cancer Center (Matsuyama, Japan) and conducted in accordance with the World Medical Association Declaration of Helsinki.

Statistical analysis. Categorical variables were analyzed using $\chi^{2}$ and Fisher's exact tests. Continuous variables were analyzed using a Mann-Whitney test. $\mathrm{P}<0.05$ was considered to indicate a statistically significant difference. The Kaplan-Meier method was used to estimate the overall survival rate in the two groups and the log-rank test was used to compare differences in the overall survival. All statistical analyses were performed with the statistical computing software $\mathrm{R}$ ( $\mathrm{R}$ version 3.5.0; $\mathrm{R}$ Core Team). 
A

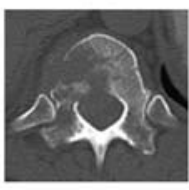

B
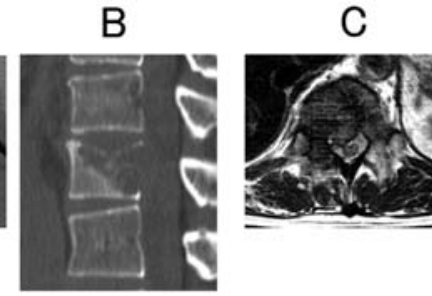

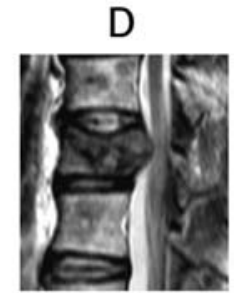

Figure 1. Malignant spinal cord compression. (A and B) Destruction of the cortex of the spinal cord column observed by computed tomography. (C and D) Malignant spinal cord compression observed by magnetic resonance imaging.

\section{Results}

MSCC prior to the establishment of the multidisciplinary treatment system. MSCC occurred in 38 patients in group A (Tables I and SII). The number of patients with neurological deficits in each of the 5 years was $8,7,6,10$ and 7 , respectively. The primary tumor sites were breast (12 patients), lung (8 patients), prostate ( 8 patients), colorectum (4 patients), stomach (4 patients), bile duct (1 patient) and multiple myeloma (1 patient). The affected locations in the spine were the cervical spine (1 patient), thoracic spine (30 patients) and lumbar spine (7 patients). The severity of the neurological deficit was Frankel A, B, C and D for 1, 1, 13 and 23 patients, respectively (Table SII). Furthermore, one patient underwent surgery following RT, whereas the other patients received RT only.

MSCC after the establishment of the multidisciplinary treatment system. MSCC occurred in 7 patients in group B (Tables I and SII). The number of patients with neurological deficits in each of the five years was $0,2,1,2$ and 2, respectively. The primary tumor sites were lung (4 patients) and breast ( 3 patients). The affected locations in the spine were the cervical spine ( 1 patient) and thoracic spine ( 6 patients). The severity of neurological deficit was Frankel C and D for 3 and 4 patients, respectively (Table SII). All patients from group B received $\mathrm{RT}$.

Comparison between the number and severity of neurological deficits before and after the establishment of the multidisciplinary treatment system. Among the patients who received RT, the number of patients with neurological deficits was $38 / 286(13.2 \%)$ and $7 / 206(3.4 \%)$ before and after the system was introduced, respectively $(\mathrm{P}<0.01)$. In patients with breast cancer, the rate of neurological deficits was higher in group $\mathrm{A}$ $(12 / 67 ; 18 \%)$ compared with in group B $(3 / 46 ; 6.5 \%)$; however, the difference was not identified to be significant $(\mathrm{P}=0.08)$. In patients with prostate cancer, the rate of neurological deficits was significantly higher in group A $(8 / 27 ; 30 \%)$ compared with group $\mathrm{B}(0 / 34 ; 0 \% ; \mathrm{P}<0.01)$. In patients with cancer of the digestive organs, the rate of neurological deficits was significantly higher in group A $(9 / 63 ; 14 \%)$ compared with group $\mathrm{B}(0 / 34 ; 0 \% ; \mathrm{P}=0.02)$. In patients with lung cancer, the rate of neurological deficits was higher in group $\mathrm{A}(8 / 97 ; 8.2 \%)$ compared with group B $(4 / 65 ; 6.2 \%)$, although the difference was not identified to be significant $(\mathrm{P}=0.62)$ (Table $\mathrm{SI})$.
Table I. Characteristics of patients with bone metastases included in this study.

\begin{tabular}{lcc}
\hline Characteristic & $\begin{array}{c}\text { Group A, } \\
\mathrm{n}(\mathrm{n}=38)\end{array}$ & $\begin{array}{c}\text { Group B, } \\
\mathrm{n}(\mathrm{n}=7)\end{array}$ \\
\hline Primary cancer site & & \\
Breast & 12 & 3 \\
Lung & 8 & 4 \\
Prostate & 8 & 0 \\
Colorectal & 4 & 0 \\
Stomach & 4 & 0 \\
Others & 2 & 0 \\
Radiation site & 1 & \\
Cervical spine & 30 & 6 \\
Thoracic spine & 7 & 0 \\
Lumbar spine & & \\
Severity of neurological & & \\
deficit (Frankel classification) & 1 & 0 \\
A & 1 & 0 \\
B & 13 & 3 \\
C & 23 & 4 \\
D & & \\
\hline
\end{tabular}

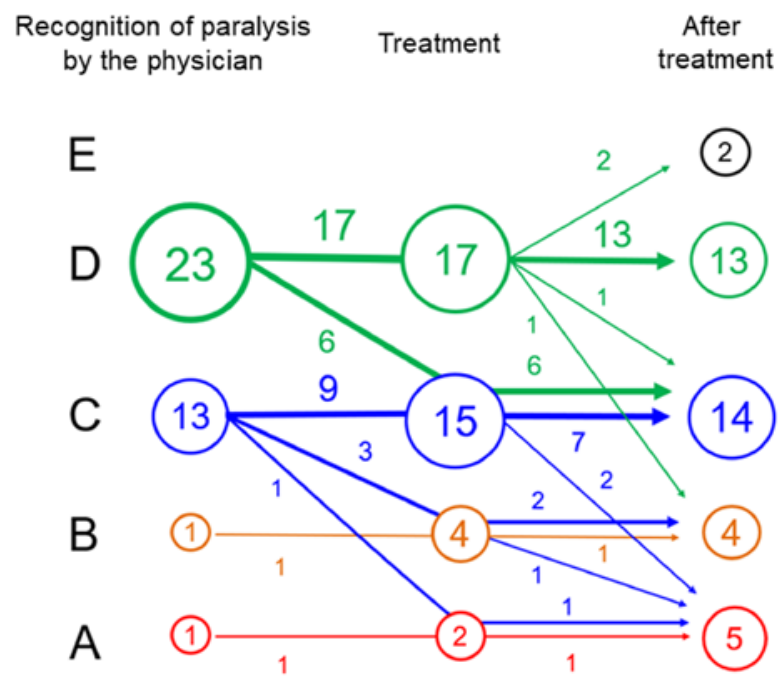

Frankel

classification

Figure 2. Severity of neurological deficit at the time of recognition by physicians, at treatment and after treatment in all patients before the establishment of the system. Improvement in the severity of neurological deficit could be attained in 2 patients $(5.3 \%)$ after treatment compared with during treatment.

In group $\mathrm{A}$, the severity of neurological deficits was Frankel A, B, C and D for 1, 1, 13 and 23 patients, respectively, at the time of their recognition by physicians (Fig. 2; Table SII). In 10/38 patients (26\%), the severity of neurological deficit was worse at the time of treatment. Following treatment, improvement in the severity of neurological deficit was achieved for 2 patients (5.3\%) with Frankel D, but not in patients with Frankel A, B or C. In patients with breast cancer, 


\section{Group A}

\section{Breast cancer}
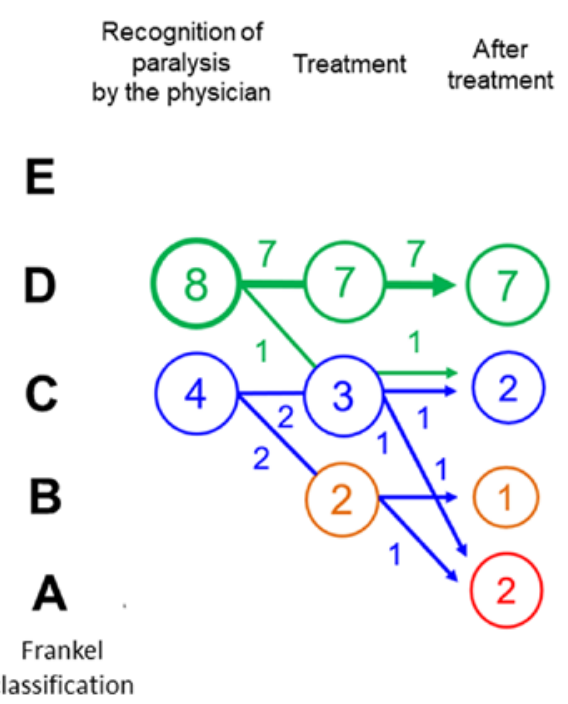

\section{Lung cancer}
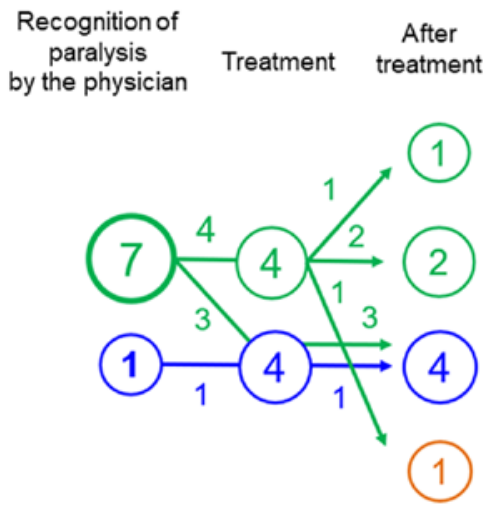

Figure 3. Severity of neurological deficit at the time of recognition by physicians, at treatment and after treatment in patients with breast and lung cancer prior to the establishment of the system. In patients with breast cancer, no patient experienced improvement in the severity of neurological deficit after treatment. In patients with lung cancer, improvement in the severity of neurological deficit was attained in 1 patient (12.5\%) prior to the establishment of the treatment system.

the severity of neurological deficit was Frankel C and D for 4 and 8 patients, respectively, at the time of recognition by physicians. In $3 / 12$ patients $(25 \%)$, the severity of neurological deficit was worse at the time of treatment. After treatment, no patient attained improvement in the severity of neurological deficit (Fig. 3). In patients with lung cancer, the severity of neurological deficit was Frankel C and D in 1 and 7 patients, respectively, at the time of recognition by physicians. In $3 / 8$ patients $(38 \%)$, the severity of neurological deficit was worse at the time of treatment. Improvement in the severity of neurological deficit after treatment was attained in 1 patient (12.5\%) with Frankel D (Fig. 3).

In group $\mathrm{B}$, the severity of neurological deficit was Frankel C and D in 3 and 4 patients, respectively, at the time of recognition by physicians. No severe neurological deficits (Frankel A and B) and no deterioration of neurological deficits were observed at the time of treatment. After treatment, improvement in the severity of neurological deficit was achieved by 2 patients (28.6\%; Fig. 4). In patients with breast cancer, the severity of neurological deficit was Frankel C and D in 1 and 2 patients, respectively, at the time of recognition by physicians. Improvement of neurological deficits was attained in 2 patients after treatment (66.7\%; Fig. 5). In patients with lung cancer, the severity of neurological deficit was Frankel C and D in 2 patients each at the time of recognition by physicians. No patient achieved improvement in the severity of neurological deficits after treatment (Fig. 5).

The rate of patients with improvement in the severity of neurological deficit after treatment was significantly increased in all patients (group A, 5.3\%; group B, 28.5\%; $\mathrm{P}<0.001$ ) and in patients with breast cancer (group A, 0\%; group B, 66.7\%; $\mathrm{P}<0.01$ ), but not in patients with lung cancer (group A, $12.5 \%$; group $\mathrm{B}, 0 \% ; \mathrm{P}=0.46$ ) following the establishment of the system.

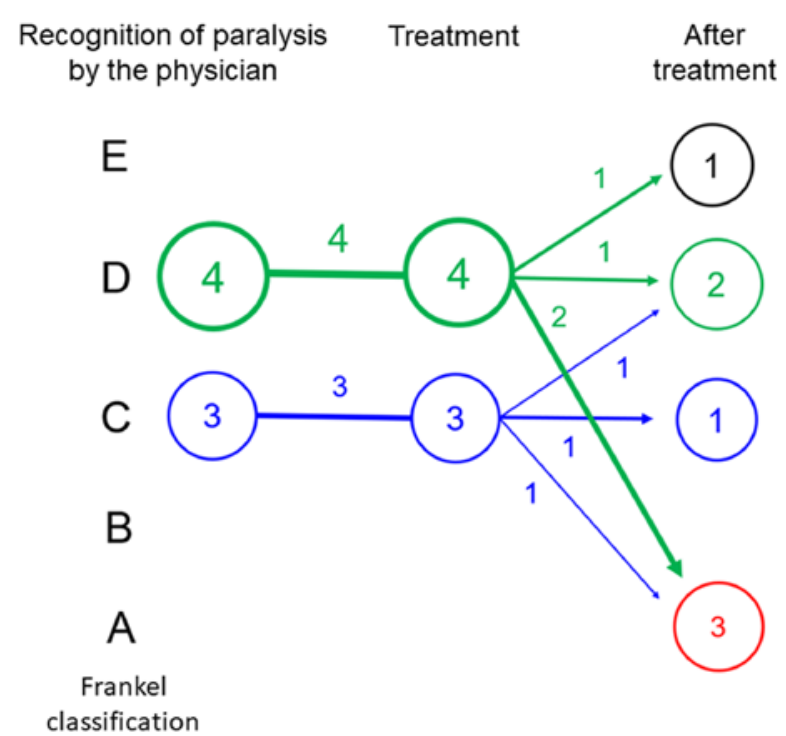

Figure 4. Severity of neurological deficit at the time of recognition by physicians, at treatment and after the treatment in all patients after the establishment of the system. Improvement in the severity of neurological deficit was attained in 2 patients $(28.6 \%)$ after treatment.

MRI scanning. The interval between the recognition of neurological deficits by physicians and MRI scanning was 0-23 days (median, 3 days) in group A (Fig. 6, Table SII). MRI was not performed in 2 patients. The interval between the recognition of neurological deficits by physicians and MRI scanning was 0 days in 7 patients, 1-3 days in 13 patients, 4-7 days in 8 patients and $>7$ days in 8 patients. The interval between the recognition of neurological deficits by physicians and MRI scanning was 0-1 days (median, 0 days) in group B. MRI was performed on the same day as the recognition of neurological 


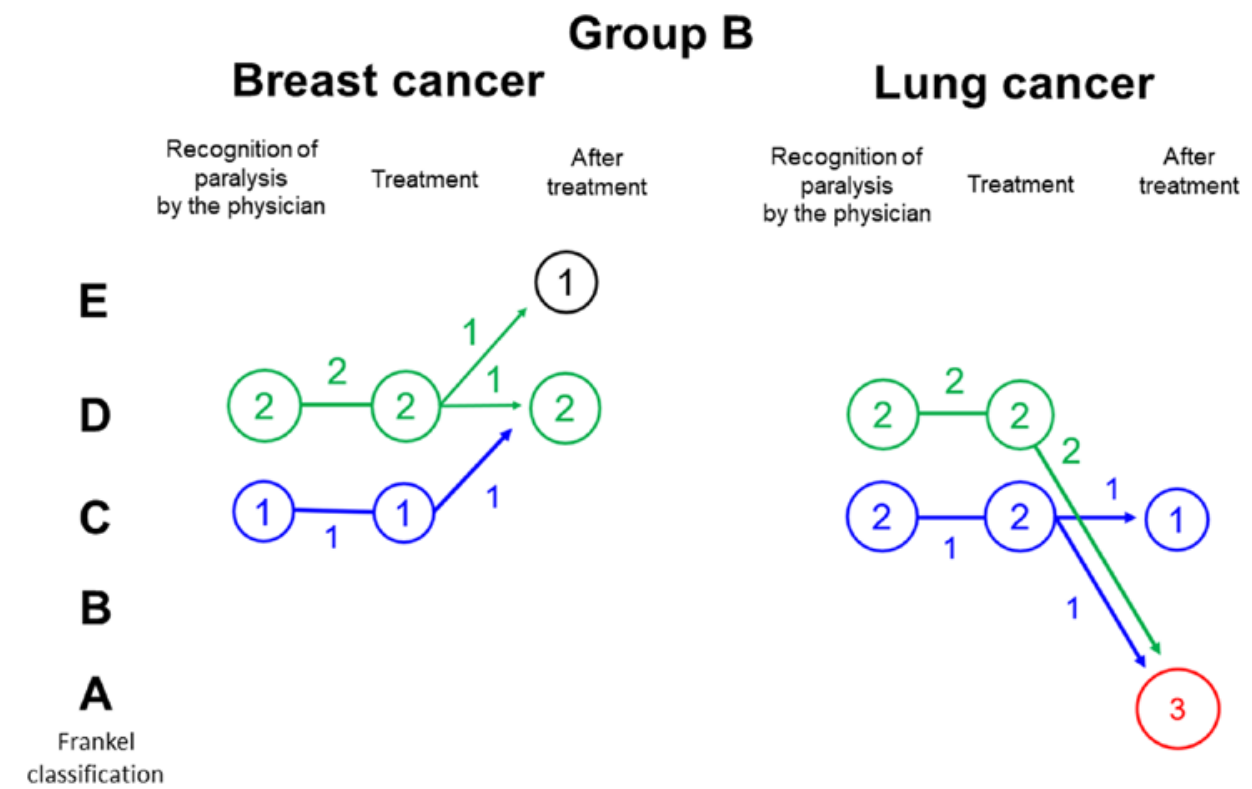

Figure 5. Severity of neurological deficit at the time of recognition by physicians, at treatment and after treatment in patients with breast and lung cancer after the establishment of the system. In patients with breast cancer, improvement in the severity of neurological deficit was attained in 2 patients (66.7\%) after the establishment of the system. In patients with lung cancer, no patient attained improvement in the severity of neurological deficit after treatment.

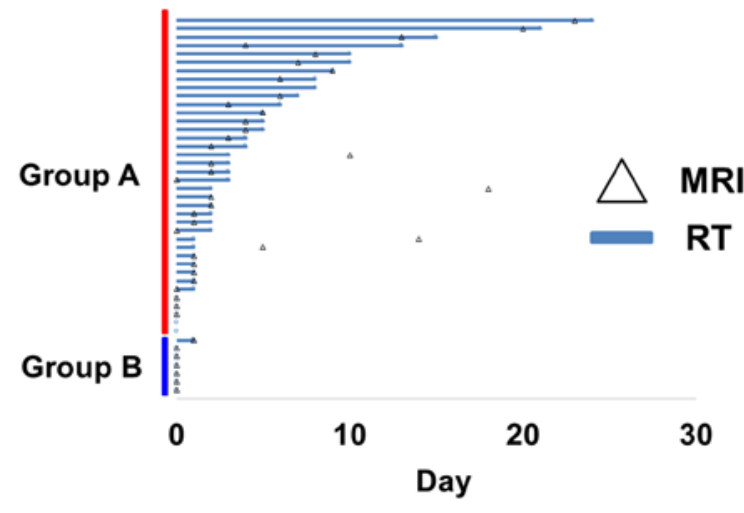

Figure 6. Time intervals between the recognition of neurological deficits by physicians, MRI scanning and treatment. The time intervals were significantly decreased after the establishment of the treatment system of bone metastases $(\mathrm{P}<0.01)$.

deficits by physicians in 5 patients $(71 \%)$ in group B. The interval between the recognition of neurological deficits by physicians and MRI scanning was significantly decreased after the establishment of the system $(\mathrm{P}<0.01$; Table SI).

Treatment initiation. The interval between the recognition of neurological deficits by physicians and the start of treatment was 0-24 days (median, 3 days) in group A (Table SII). The interval between the recognition of neurological deficits by physicians and the start of treatment was 0 days in 5 patients, 1-3 days in 17 patients, 4-7 days in 7 patients, and $>7$ days in 9 patients (Table SII). The interval between the recognition of neurological deficits by physicians and the start of treatment was 0 -1 days (median, 0 days) in group B (Table SII). Treatment was provided on the same day in all but 2 patients in group $\mathrm{B}$. The interval between the recognition of neurological deficits by physicians and treatment was significantly decreased after the establishment of the system $(\mathrm{P}<0.01)$ (Table SII).

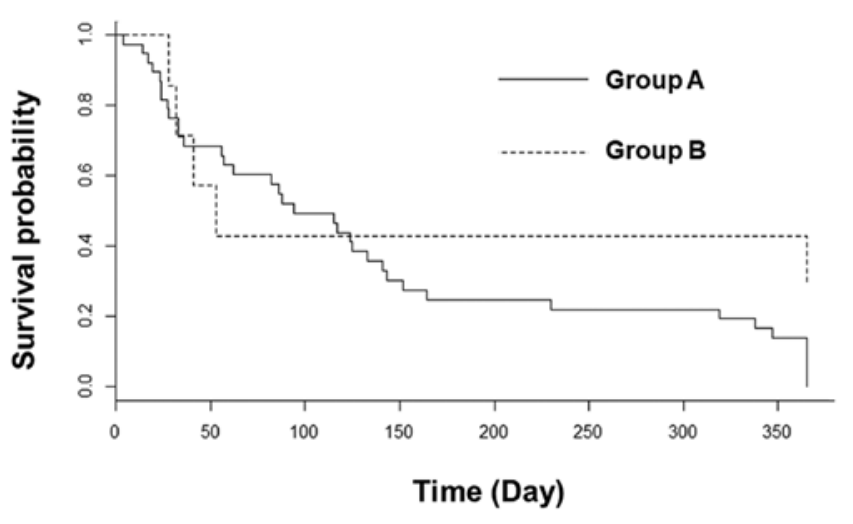

Figure 7. Overall survival of groups A and B estimated by the Kaplan-Meier method, with no significant difference being observed $(\mathrm{P}=0.36)$.

Referral to orthopedists. The rates of referral to orthopedists before treatment in groups A and B were 17/38 (45\%) and $7 / 7(100 \%)$, respectively $(\mathrm{P}<0.01)$ (Table SII). The use of BMAs before the recognition of neurological deficits by physicians was 15/38 (39\%) and 7/7 (100\%) in groups A and B, respectively $(\mathrm{P}<0.01)$ (Table SII). BMAs were administered at the time of diagnosis of bone metastases to all patients in group B.

Overall survival rates. At the last follow-up, $37 / 38$ patients and 5/7 patients had died in groups A and B, respectively. The overall survival rates were 24 and $43 \%$ in groups A and B, respectively, at 6 months following RT with no significant difference identified $(\mathrm{P}=0.36$; Fig. 7).

\section{Discussion}

The present study reported a significant improvement in the rate of neurological deficit cases when using a multidisciplinary treatment system for patients with bone metastases. 
Furthermore, the rate of patients with improvement in the severity of neurological deficit after treatment was improved after the establishment of the system.

An innovative treatment approach, including intensity-modulated RT, vertebroplasty and minimally invasive surgery, has been developed (21). However, improvement of the neurological deficit is difficult once it occurs. Therefore, early diagnosis, treatment and prevention of MSCC are crucial in order to minimize treatment delay. In numerous cases, a neurological deficit does not occur suddenly, and back pain appears as a prodromal symptom for several weeks prior. Therefore, several guidelines for bone metastases recommend that clinicians watch for this early sign of MSCC and provide an emergency diagnosis by using spine MRI $(5,10)$. Furthermore, the occurrence of MSCC should be considered when back pain appears in a patient with spinal metastases (so-called 'Red Flag'), and emergency imaging, including CT or MRI, should be performed. When imaging cannot be performed immediately, the most recent imaging should be reviewed, and the presence of vertebral body collapse or asymptomatic MSCC should be confirmed. If treatment is initiated before a neurological deficit fully develops, it can be prevented in certain patients (preventable paralysis).

In the present study, the incidence of paralysis decreased following the establishment of the system. The rate of patients with neurological deficit and the rate of patients with improvement in the severity of neurological deficit after treatment were significantly improved. In addition, among patients who received RT, the rate of patients with neurological deficit was $38 / 286(13.2 \%)$ and $7 / 206$ (3.4\%) before and after the establishment of the system, respectively, and this difference was identified to be significant. Although the rate of patients with neurological deficit was lower in all types of cancer, there was no significant difference observed between patients with breast and lung cancer.

Numerous patients presented with severe levels of neurological deficit (Frankel A and B), and the severity of neurological deficit was worse at the time of treatment in $26 \%$ of patients before the establishment of the system. However, no severe level of neurological deficit and no deterioration of the neurological deficit were seen at the time of treatment after the establishment of the system.

The rate of patients with improvement in the severity of neurological deficit was 5.3 and $28.5 \%$ before and after the establishment of the system, respectively, which was significantly different. Urgent treatment with the system could lead to the prevention of the deterioration of the neurological deficit at the time of treatment and improvement of the neurological deficit.

The rate of patients with improvement in the severity of neurological deficit after treatment was significantly improved in patients with breast cancer, but not in patients with lung cancer, after the establishment of the system. This can be explained by the difference in the sensitivity to RT between patients with breast and lung cancer. Since breast cancer is more sensitive to RT (4), early treatment may lead to an improvement in the severity of neurological deficits.

It has been hypothesized that the increased awareness of back pain as a sign of MSCC and subsequent earlier referral to orthopedists could lead to the early diagnosis and treatment of MSCC. Additionally, the system depends on the capacity of radiologists to identify patients with vertebral body collapse who may require a potential prophylactic approach to prevent neurological deficit.

Previous studies have demonstrated that specific systems developed for earlier diagnosis and treatment can decrease treatment delays, which can lead to improved neurological outcomes of patients $(11,18)$. Savage et al $(11)$ reported the establishment of a rapid referral system designed to provide urgent access to MRI scanning and urgent referral to oncology specialists for the management of MSCC. Key steps in the management of patients with suspicion of MSCC would include the capacity of physicians to directly connect with the oncology specialist in a tertiary center in order to provide access to urgent MRI scanning for patients. Furthermore, physicians can provide an alert card to the patients with high risk of MSCC in order to encourage self-referral to the hospital when back pain, which is a potential symptom of MSCC, occurs. By using this approach, it has been reported that the rate of patients with major impairment decreased from 80 to $62 \%$ (11). Allan et al (18) reported a similar system for improving the time to diagnosis via a hotline by which physicians can directly connect with the oncology specialist in a tertiary center for patients with suspicion of MSCC and in urgent need for MRI scanning. Using this approach, it has been reported that the time to MRI is shortened, and that the proportion of patients who are unable to walk at MRI decreases from 46 to $23 \%$ (18). These results suggest that the establishment of such a system is effective in daily clinical practice and that it can improve the time to referral.

Furthermore, the present study reported a significant decrease in the time from the recognition of a neurological deficit by physicians to MRI scanning and treatment after the establishment of the treatment system for bone metastases. Approximately $40 \%$ of patients underwent MRI at $>3$ days following recognition of a neurological deficit by physicians, and in $26 \%$ of the patients, the severity of the neurological deficit was worse at the time of the treatment before the system was implemented. The rate of referral to orthopedists before treatment was only $45 \%$. Conversely, all patients were referred to orthopedists, $71 \%$ of patients underwent MRI and RT on the same day, and the remaining $29 \%$ of patients underwent MRI and RT the day after the recognition of neurological deficit by physicians after the system was implemented.

The use of BMAs was also increased following the establishment of the system. Although the use of BMAs before the recognition of a neurological deficit by physicians was only $39 \%$ in patients before the establishment of the system, they were administered at the time of diagnosis of bone metastases in all patients after the establishment of the system. This could be due to the increased efforts of physicians to treat patients with bone metastases.

A novel multidisciplinary treatment system is required to establish an effective and intensive diagnosis and treatment system for patients with bone metastases (19). Hirai et al (17) demonstrated that early diagnosis through surveillance of bone metastases in patients with hepatocellular carcinoma can prevent fractures and paralysis, which can lead to an improved quality of life. Recently, cancer boards, including 
teams of doctors specialized in orthopedy, oncology, palliative care, radiotherapy and radiology, nurses, physical therapists, occupational therapists and medical social workers, focusing on the management of bone metastases were organized in a number of institutions in Japan (19). In certain institutions, radiologists review all imaging studies of patients with bone metastases and can, thus, identify patients that may require a prophylactic approach to prevent paralysis (18). With this system, a common understanding of the importance of managing bone metastases is established among all members of the cancer board.

Although the multidisciplinary treatment system is effective for the management of bone metastases, this system presents some limitations. With this system, it can be difficult to identify patients with imminent development of MSCC in a timely manner. For example, in certain patients with osteoblastic bone metastases and extravertebral metastases, it can be hard for the radiologist to identify MSCC by routine CT alone. Furthermore, physicians can occasionally overlook signs of MSCC, including back pain, motor dysfunction and sensory disturbance if they are not severe. In addition, patients do not always mention the back pain to physicians or do not come to the hospital when lower leg weakness is not severe. There is currently no system in The Shikoku Cancer Center (Matsuyama, Japan) that could be used to inform or educate patients about back pain as an early warning sign of MSCC, and alert them to go to hospital earlier. Therefore, neurological deficits cannot be prevented in some patients. The rate of early diagnosis and prevention of potential neurological deficits could be improved if patients with bone metastases had been informed about the importance of contacting physicians when complaining of back pain, especially when the pain is accompanied by neurological signs. Since MSCC occurs in only a small percentage of patients with bone metastases, it may be reasonable to select patients at high risk for education.

Another limitation of the system is the variable background of patients with bone metastases. Since patients presented with various types of primary tumor, metastatic sites, systemic therapies and general status at the time of the neurological deficit caused by MSCC, the individual prognoses were different. These factors could be responsible for the absence of a significant difference in the overall survival rates before and after the establishment of the system presented in the present study. However, these limitations are common in the study of bone metastases.

In conclusion, the present study demonstrated that the times between the recognition of a neurological deficit by physicians to MRI scanning and the treatment were significantly decreased after the establishment of the multidisciplinary treatment system for bone metastases. In addition, no deterioration of neurological deficits was observed at the time of treatment after the establishment of the multidisciplinary treatment system. Furthermore, the rate of patients with improvement in the severity of neurological deficits after treatment was significantly increased. The establishment of a multidisciplinary treatment system for patients with bone metastases may therefore be useful for the early diagnosis and treatment and prevention of MSCC in patients with bone metastases.

\section{Acknowledgements}

Not applicable.

\section{Funding}

No funding was received.

\section{Availability of data and materials}

The datasets used and/or analyzed during the current study are available from the corresponding author on reasonable request.

\section{Authors' contributions}

EN, KN, TF, TT and YA designed the study, and collected and analyzed data. RN, TK and TO analyzed data. YS and SS treated the patients presented in this manuscript and collected and analyzed data. All authors read and approved the final manuscript.

\section{Ethics approval and consent to participate}

The present study was approved by the Ethics Committee of Shikoku Cancer Center (Matsuyama, Japan).

\section{Patient consent for publication}

Not applicable.

\section{Competing interests}

The authors declare that they have no competing interests.

\section{References}

1. Harel R and Angelov L: Spine metastases: Current treatments and future directions. Eur J Cancer 46: 2696-2707, 2010.

2. Sciubba DM, Petteys RJ, Dekutoski MB, Fisher CG, Fehlings MG, Ondra SL, Rhines LD and Gokaslan ZL: Diagnosis and management of metastatic spine disease. A review. J Neurosurg Spine 13: 94-108, 2010

3. Nakata E, Sugihara S, Kataoka M, Yamashita N, Furumatsu T, Takigawa T, Tetsunaga T and Ozaki T: Early response assessment of palliative conventional radiotherapy for painful uncomplicated vertebral bone metastases. J Orthop Sci 23: 912-917, 2018

4. Nakata E, Sugihara S, Kataoka M, Yamashita N, Furumatsu T, Takigawa T, Tetsunaga T and Ozaki T: Early response assessment of re-ossification after palliative conventional radiotherapy for vertebral bone metastases. J Orthop Sci 24: 332-336, 2019.

5. White BD, Stirling AJ, Paterson E, Asquith-Coe K and Melder A; Guideline Development Group: Diagnosis and management of patients at risk of or with metastatic spinal cord compression: Summary of NICE guidance. BMJ 337: a2538, 2008.

6. Solberg A and Bremnes RM: Metastatic spinal cord compression: Diagnostic delay, treatment, and outcome. Anticancer Res 19: 677-684, 1999.

7. Shibata H, Kato S, Sekine I, Abe K, Araki N, Iguchi H, Izumi T, Inaba Y, Osaka I, Kato S, et al: Diagnosis and treatment of bone metastasis: Comprehensive guideline of the Japanese Society of Medical Oncology, Japanese Orthopedic Association, Japanese Urological Association, and Japanese Society for Radiation Oncology. ESMO Open 16: e000037, 2016.

8. Levack P, Graham J, Collie D, Grant R, Kidd J, Kunkler I, Gibson A, Hurman D, McMillan N, Rampling R, et al: Don't wait for a sensory level-listen to the symptoms: A prospective audit of the delays in diagnosis of malignant cord compression. Clin Oncol (R Coll Radiol) 14: 472-480, 2002. 
9. Tsukada Y, Nakamura N, Ohde S, Akahane K, Sekiguchi K and Terahara A: Factors that delay treatment of symptomatic metastatic extradural spinal cord compression. J Palliat Med 18: $107-113,2015$

10. Loblaw DA, Mitera G, Ford M and Laperriere NJ: A 2011 updated systematic review and clinical practice guideline for the management of malignant extradural spinal cord compression. Int J Radiat Oncol Biol Phys 84: 312-317, 2012.

11. Savage P, Sharkey R, Kua T, Schofield L, Richardson D, Panchmatia N, Papanastasopoulos P, Williams M, Falconer A, Power D, et al: Malignant spinal cord compression: NICE guidance, improvements and challenges. QJM 107: 277-282, 2014.

12. Vieillard $\mathrm{MH}$ and Thureau S: Multidisciplinary meetings dedicated to bone metastases: A historical perspective and rationale. Bull Cancer 100: 1135-1139, 2013 (In French).

13. Blum RH, Novetsky D, Shasha D and Fleishman S: The multidisciplinary approach to bone metastases. Oncology (Williston Park) 17: 845-857; discussion 862-863, 867, 2003.

14. Ibrahim T, Flamini E, Fabbri L, Serra P, Mercatali L, Ricci R, SacannaE,Falasconi MC,CasadeiR,GalassiR, et al:Multidisciplinary approach to the treatment of bone metastases: Osteo-Oncology Center, a new organizational model: Tumori 95: 291-297, 2009.

15. Thavarajah N, Wong K, Zhang L, Bedard G, Wong E, Tsao M, Danjoux C, Barnes E, Sahgal A, Dennis K, et al: Continued success in providing timely palliative radiation therapy at the Rapid Response Radiotherapy Program: A review of 2008-2012. Curr Oncol 20: e206-e211, 2013.

16. Bongiovanni A, Recine F, Fausti V, Foca F, Casadei R, Falasconi MC, Oboldi D, Sansoni E, Fabbri L, Micheletti S, et al: Ten-year experience of the multidisciplinary Osteoncology Center. Support Care Cancer 27: 3395-3402, 2019.
17. Hirai T, Shinoda Y, Tateishi R, Asaoka Y, Uchino K, Wake T, Kobayashi H, Ikegami M, Sawada R, Haga N, et al: Early detection of bone metastases of hepatocellular carcinoma reduces bone fracture and paralysis. Jpn J Clin Oncol 49: 529-536, 2019.

18. Allan L, Baker L, Dewar J, Eljamel S, Grant RM, Houston JG, McLeay T, Munro AJ and Levack P: Suspected malignant cord compression-improving time to diagnosis via a 'hotline': A prospective audit. Br J Cancer 100: 1867-1872, 2009.

19. Kimura T: Multidisciplinary approach for bone metastasis: A review. Cancers (Basel) 10: pii: E156 2018.

20. Frankel HL, Hancock DO, Hyslop G, Melzak J, Michaelis LS, Ungar GH, Vernon JD and Walsh JJ: The value of postural reduction in the initial management of closed injuries of the spine with paraplegia and tetraplegia. I. Paraplegia 7: 179-192, 1969.

21. Ejima Y, Matsuo Y and Sasaki R: The current status and future of radiotherapy for spinal bone metastases. J Orthop Sci 20: $585-592,2015$.

This work is licensed under a Creative Commons Attribution-NonCommercial-NoDerivatives 4.0 International (CC BY-NC-ND 4.0) License. 